\section{Tamping down TAU}

\section{By Lauren Martz, Staff Writer}

Despite evidence that pathologic TAU spreads through the brain in tauopathies such as Alzheimer's disease and frontotemporal dementia, the actual mechanism by which the misfolded propagates is murky. A key player in the process is heparan sulfate proteoglycans, according to new findings from the Washington University in St. Louis School of Medicine.

These results suggest that heparan sulfate proteoglycans (HSPGs) are responsible for neuronal uptake of misfolded TAU (microtubuleassociated protein- $\tau$; MAPT; FTDP-17) and that inhibiting HSPGs could prevent or slow progression of tauopathies. The findings add tauopathies to the list of diseases in which HSPGs are involved and add the glycoproteins as new targets for companies tackling tauopathies. The challenge will be safely hitting the target because it is widely distributed.

HSPGs are cell surface glycoproteins found on all healthy cells that bind a wide variety of ligands. In addition to important functions in embryonic development, the molecules are involved in the normal functioning of organ systems, with cell-signaling roles in processes ranging from metabolism to injury repair.

Initial hints that the molecules had a role in disease date back to the 1983 finding that overexpression of an enzyme that degrades HSPGs correlated with invasion and metastasis in a mouse model of melanoma. ${ }^{2}$ The glycoproteins also have been implicated in inflammatory diseases.

In 2005, researchers found that cell surface HSPGs were responsible for the uptake of misfolded infectious extracellular prions. Once inside healthy cells, the prions can trigger the misfolding and export of native prions, propagating the spread of neurodegeneration. ${ }^{3}$

Those findings collectively caught the eye of Marc Diamond, a professor of neurology at the Washington University in St. Louis School of Medicine, who hypothesized that HSPGs may be at play in tauopathies. Tauopathies involve the conversion of native TAU into insoluble, aggregation-prone fibrils that lead to neuronal cell death.

In 2009, Diamond and colleagues described the spread of TAU pathology as a prion-like transcellular propagation. ${ }^{4}$ The team found that when TAU aggregates are internalized by normal cells, they can trigger the fibrillization of native TAU.

Last year, the group found that TAU aggregates are released from cells into the extracellular space, allowing the uptake and spread of the misfolded aggregates through the brain. ${ }^{5}$
Now, Diamond and colleagues have found that TAU fibrils-like prion proteins-are internalized by HSPGs. The team included researchers from the University of California, Berkeley School of Optometry, Paris 12 Val de Marne University and the University of California, San Francisco.

In neuronal precursor cells, TAU repeat domains prone to aggregation specifically bound HSPGs on cell surfaces and were internalized. The HSPG inhibitors heparin and chlorate prevented the process. Heparin competitively inhibits HSPGs, whereas chlorate prevents the sulfation of HSPGs that is required for ligand binding.

In neuronal precursor cells cultured with TAU repeat domain fibrils, pretreatment with heparinase III, which degrades HSPGs, decreased the percentage of TAU aggregate-expressing cells as well as the number of aggregates per cell compared with pretreatment using a control enzyme.

The findings extended to mature neurons and full-length TAU.

In primary hippocampal neurons, small hairpin RNA targeting exostoses 1 (EXT1), an enzyme required for HSPG synthesis, decreased TAU fibril uptake compared with a control shRNA.

In an assay using human embryonic kidney cells, coculture of cells expressing misfolded, disease-mutant TAU with cells expressing wild-type TAU caused the protein to aggregate in the healthy cells. This effect was blocked by HSPG inhibitors. The assay was not possible in neuronal cells.

The researchers also co-injected TAU fibrils with either a heparin mimetic or control mimetic into the brains of mice. Neurons from the cortices of mice treated with the heparin mimetic had fewer TAU fibrils than neurons from mice treated with the control mimetic, indicating that the heparin mimetic blocked uptake of the fibrils.

Finally, the team showed that internalization and aggregation of $\alpha$-synuclein (SNCA) into neuronal precursor cells was also blocked by chlorate or heparin. That result suggested that synucleinopathies propagate with a mechanism similar to that for tauopathies.

Data were published in the Proceedings of the National Academy of Sciences.

"This was a great first step, and the data in non-neuronal cells were rock solid, but next they should do more work in real neurons with full-length TAU," said George Bloom, a professor of biology and cell biology at the University of Virginia. "There are

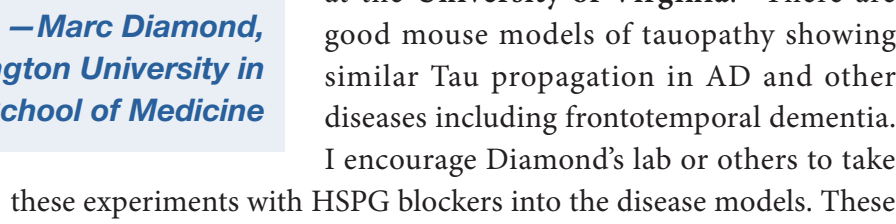
\begin{tabular}{c|c}
-Marc Diamond, & $\begin{array}{l}\text { good mouse models of tauopathy showing } \\
\text { similar Tau propagation in AD and other } \\
\text { diseases including frontotemporal dementia. }\end{array}$ \\
I encourage Diamond's lab or others to take
\end{tabular} \begin{tabular}{c|c}
-Marc Diamond, & $\begin{array}{l}\text { good mouse models of tauopathy showing } \\
\text { similar Tau propagation in AD and other } \\
\text { diseases including frontotemporal dementia. }\end{array}$ \\
I encourage Diamond's lab or others to take
\end{tabular} \begin{tabular}{c|c}
-Marc Diamond, & $\begin{array}{l}\text { good mouse models of tauopathy showing } \\
\text { similar Tau propagation in AD and other } \\
\text { diseases including frontotemporal dementia. }\end{array}$ \\
I encourage Diamond's lab or others to take
\end{tabular} data could determine if the approach slows or stops disease progression and could begin to assess adverse effects."

Diamond said that his team has "other plans to test this pathway further in animal models."

Interaction inhibition

Diamond said that his team is developing small molecules that block 


\section{ANALYSIS}

\section{TARGETS \& MECHANISMS}

the TAU and HSPG interactions. A key challenge will be restricting exposure of the molecules to the brain.

"One problem in the development of HSPG inhibitors may be that you disturb a normal activity that has a biological role," noted Christer Möller, CSO of BioArctic Neuroscience AB. BioArctic and partner Eisai Co. Ltd. are developing BAN2401, a humanized antibody targeting toxic $\beta$-amyloid (A $\beta$ ) protofibrils. The compound is in Phase II testing to treat $\mathrm{AD}$.

Bloom added, "HSPG is in every cell and all over the body. Anything that interferes with the interaction between HSPGs and TAU fibrils runs the risk of interfering with other HSPG interactions. Developing this type of therapeutic is akin to developing a new chemotherapy for cancer. We hope that cancer cells will be more sensitive than the healthy cells, but we know that the healthy cells will be adversely affected to some extent."

Diamond agreed that there are multiple potential side effects that will need to be assessed or avoided. "The one I am most aware of is bone growth abnormalities. Obviously, these compounds could also disrupt developmental processes, but with luck, this won't be an issue in the aged population," he said.

His team also is working to determine the genes in the HSPG pathway most important for TAU fibril uptake, which could allow for reduced adverse effects.

The good news, said Diamond, is that small molecule HSPG inhibitors should be able to cross the blood brain barrier. "If small molecule HSPG inhibitors could be developed, we could potentially have an oral medication that might treat tauopathy and synucleinopathy plus several other diseases," he said.

Bloom said that a therapeutic that blocks TAU aggregate propagation is only half the battle. "We will need to find an effective way to identify patients much earlier in the course of the disease," he said.

The problem, he said, is that the cognitive symptoms and diagnosis usually occur about 25 years after pathology has started, when massive and irreversible brain damage and spreading have already occurred.

Morten Albrechtsen, CEO at Enkam Pharmaceuticals A/S, agreed and said that a problem with many AD therapeutic candidates is determining whether they will be effective at preventing disease, stopping its progression or curing patients.

Enkam is developing FGLs, a synthetic peptide that allosterically modulates fibroblast growth factor receptor (FGFR). The peptide is in preclinical testing for $\mathrm{AD}$.

Diamond said that the Washington University in St. Louis has filed a patent application covering the work and that the IP is unavailable for licensing.

Martz, L. SciBX 6(32); doi:10.1038/scibx.2013.848

Published online Aug. 22, 2013

\section{REFERENCES}

1. Holmes, B.B. et al. Proc. Natl. Acad. Sci. USA; published online July 29, 2013; doi:10.1073/pnas.1301440110

Contact: Marc I. Diamond, Washington University in St. Louis School of Medicine, St. Louis, Mo. e-mail: diamondm@neuro.wustl.edu

2. Nakajima, M. et al. Science 220, 611-613 (1983)

3. Horonchik, L. et al. J. Biol. Chem. 280, 17062-17067 (2005)

4. Frost, B. et al. J. Biol. Chem. 284, 12845-12852 (2009)

5. Kfoury, N. et al. J. Biol. Chem. 287, 19440-19451 (2012)

\section{COMPANIES AND INSTITUTIONS MENTIONED}

BioArctic Neuroscience AB, Stockholm, Sweden Eisai Co. Ltd. (Tokyo:4523; Osaka:4523), Tokyo, Japan Enkam Pharmaceuticals A/S, Copenhagen, Denmark Paris 12 Val de Marne University, Creteil, France University of California, Berkeley School of Optometry, Berkeley, Calif.

University of California, San Francisco, Calif. University of Virginia, Charlottesville, Va. Washington University in St. Louis, St. Louis, Mo. Washington University in St. Louis School of Medicine, St. Louis, Mo. 